\title{
BANACH SPACES WHICH EMBED INTO THEIR DUAL
}

\author{
VALERIO CAPRARO AND STEFANO ROSSI
}

\begin{abstract}
We use Birkhoff-James' orthogonality in Banach spaces to provide new conditions for the converse of the classical Riesz's representation theorem.
\end{abstract}

\section{INTRODUCTION}

It is well-known to everyone that the two most basic properties of a complex Hilbert space $\mathcal{H}$ are

- If $X$ is a closed subspace of $\mathcal{H}$, then $\mathcal{H}=X \oplus X^{\perp}$.

- (Riesz's representation theorem) There is a conjugate-linear isometry from $\mathcal{H}$ onto $\mathcal{H}^{*}$.

It was shown by Lindenstrauss and Tzafriri in Li-Tz71 that the first property essentially characterizes Hilbert spaces among the Banach spaces. A longstanding question asks instead whether Riesz's representation theorem also characterizes the Hilbert spaces; namely, let $X$ be a Banach space and $F: X \rightarrow X^{*}$ an isometric isomorphism, is it true that $X$ is a Hilbert space? In general, the answer is clearly negative. Indeed, let $Y$ be a reflexive Banach space which is not a Hilbert space, one can easily check that $X=Y \oplus Y^{*}$ is isometrically isomorphic to its dual, but $X$ is not an Hilbert space. So, over the years, there have been many attempts to add some condition on $F$ in order to guarantee that $X$ turns to be a Hilbert space (see, for instance, Dr-Ya05, Li70, Pa86a, Sz-Za81]). In this paper we contribute to this problem proposing some different conditions, by making use of the so-called Birkhoff-James' orthogonality (see Theorems 2 and 4). We also propose some weaker statement, as in Theorem 5 and its corollary.

\section{Some converses of the Riesz Representation theorem}

Throughout this note $(X,\|\cdot\|)$ will denote a complex normed Banach space (the real case is just analogue). We start recalling Birkhoff-James' definition of orthogonality in Banach spaces (cfr. Bi35] and Ja47).

Definition 1. $x \in X$ is said to be orthogonal to $y \in X$ if for each scalar $\lambda$ one has

$$
\|x\| \leq\|x+\lambda y\|
$$

It is clear that if $X$ is an Hilbert space, then this definition reduces to the usual one. In this general context, where there is no inner product, it describes the following geometric property: a vector $x$ is orthogonal to $y$ if each triangle with one side equal to $x$ and another side constructed along $y$ has the third side longer than $x$. By the way, this is not the unique definition of orthogonality in Banach spaces, but it is surely the oldest and the most intuitive one (see [Al-Be97, [Di83], [Ja45] and $\mathrm{Pa} 86 \mathrm{~b}$ for other notions of orthogonality).

2000 Mathematics Subject Classification. Primary 52A01; Secondary 46L36.

Supported by Swiss SNF Sinergia project CRSI22-130435. 
A simple but important remark is that the classical Riesz representation $\mathcal{H} \ni x \rightarrow f_{x} \in \mathcal{H}^{*}$ verifies the property $x \in \operatorname{Ker}\left(f_{x}\right)^{\perp}$, that we can require in our context of normed spaces by using the Birkhoff-James orthogonality (by the way, it would be interesting to know if the following result holds true also using other notion of orthogonality).

Theorem 2. Let $X$ be a complex normed (resp. Banach) space and $F: x \in X \rightarrow f_{x} \in X^{*}$ an isometry such that for all $x, y \in X$ one has

$$
f_{x}(y)=\overline{f_{y}(x)}
$$

$$
x \in \operatorname{Ker}\left(f_{x}\right)^{\perp}
$$

Then $X$ is a pre-Hilbert (resp. Hilbert) space with respect to the inner product given by $(x, y)=f_{x}(y)$ and $(x, x)=\|x\|^{2}$.

Proof. Clearly $(x, y) \doteq f_{x}(y)$ defines a sesquilinear hermitian form on $\mathrm{X}$ (thanks to 1). We will prove that this form is also positive definite. Let $x \in X$ be such that $(x, x)=0$, then $x \in \operatorname{Ker}\left(f_{x}\right)$ and we can apply Definition 1 with $\lambda y=-x$ : $\|x\| \leq 0$, i.e. $x=0$. Now we observe that the realvalued function $\Phi: X \ni x \rightarrow f_{x}(x) \in \mathbb{R}$ is continuous (by triangle inequality), $X \backslash\{0\}$ is connected (unless $\operatorname{dim} X=1$ and $X$ is real, which is a trivial case) and thus $\Phi(X \backslash\{0\})$ is an interval $I \subseteq \mathbb{R}$ not containing 0 . Whence $I \subseteq(-\infty, 0)$ or $I \subseteq(0, \infty)$. This shows that it is not restrictive assume that $f_{x}(x)>0$ for each $x \neq 0$ (otherwise take $-f_{x}(x)$ ). It remains to prove that $f_{x}(x)=\|x\|^{2}$. Clearly $f_{x}(x) \leq\left\|f_{x}\right\|\|x\|=\|x\|^{2}$. Conversely, let $p(x)$ such that $f_{x}(x)=p(x)\|x\|$. We have to prove that $p(x) \geq\|x\|$. Let $y \in \operatorname{Ker}\left(f_{x}\right)$ and $\lambda \in \mathbb{C}$, by Definition 1, we have

$$
\left|f_{x}(\lambda x+y)\right|=|\lambda| f_{x}(x)=|\lambda| p(x)|| x||=p(x)\|\lambda x|| \leq p(x)\| \lambda x+y \|
$$

Now, remember that when $y$ runs over $\operatorname{Ker}\left(f_{x}\right)$ and $\lambda \in \mathbb{C}, \lambda x+y$ describes the whole $X$ (Indeed $\operatorname{Ker}\left(f_{x}\right)$ has codimension 1 and does not contain $\left.x\right)$, whence $\|x\|=\left\|f_{x}\right\| \leq p(x)$.

Remark 3. In Pa86a the author proved that the existence of an orthogonality relation on a Banach space which satisfies certain properties is sufficient to guarantee that the Banach space is actually a Hilbert space. Unfortunately, the Birkhoff-James orthogonality does not satisfy those properties. In [Dr-Ya05], Proposition 3.1 of the version in the arXiv, the authors proved a result similar to our Theorem Q Indeed they obtained the same conclusion under the conditions $f_{x}(x) \geq 0$ for all $x \in X$ and $x \rightarrow f_{x}$ surjective. Their first condition is then weaker than ours; while the second one is stronger. Indeed, we have not required that $F: X \rightarrow X^{*}$ is surjective, being a consequence of the other hypothesis, at least when $X$ is a norm-complete. In fact, requiring surjectivity a priori, we are able to relax our second hypothesis.

Theorem 4. Let $F: x \in X \rightarrow f_{x} \in X^{*}$ be an isometric isomorphism that satisfies the following

(1) $f_{x}(y)=\overline{f_{y}(x)}$

(2) $x \in \operatorname{Ker}\left(f_{x}\right) \Rightarrow x=0$

Then $X$ is a Hilbert space with respect to the inner product given by $(x, y)=f_{x}(y)$ and $(x, x)=\|x\|^{2}$.

Proof. The same argument of the previous proof shows that $(\cdot, \cdot)$ is positive definite. Setting $|x|=$ $(x, x)^{1 / 2}$, it remains only to prove that $|x|=\|x\|$, for each $x \in X$. Clearly $|x|^{2} \leq\left\|f_{x}\right\| \mid x\|=\| x \|^{2}$. Conversely, by the Hahn-Banach theorem, there exists $f \in X^{*}$, with $\|f\|=1$, such that $\|x\|=f(x)$. 
By the surjectivity of the embedding we have $f=f_{y}$, for some $y \in X$ with $\|y\|=\left\|f_{y}\right\|=\|f\|=1$. So

$$
\|x\|=f_{y}(x)=(y, x) \leq|y||x| \leq|| y|| \cdot|x|=|x|
$$

in which the first inequality is nothing but the Cauchy-Schwarz inequality applied to $(\cdot, \cdot)$.

Now we propose a little refinement of the previous results. Indeed, if $X$ is reflexive and $R a n F$ is closed we get the same conclusion, up to norm-equivalence. More precisely

Theorem 5. Let $F: x \in X \rightarrow f_{x} \in X^{*}$ be a continuous map from the reflexive Banach space $X$ into its dual with closed range and such that

$$
\begin{gathered}
f_{x}(y)=\overline{f_{y}(x)} \\
x \in \operatorname{Ker}\left(f_{x}\right) \Rightarrow x=0
\end{gathered}
$$

Then the norm of $X$ is equivalent to the Hilbert norm given by $|x|=f_{x}(x)^{1 / 2}$.

Proof. We start observing that $F$ is injective (by 4), so it is an isomorphism between $X$ and RanF. Then, by the Banach inverse operator theorem we get $\left\|f_{x}\right\| \geq \delta\|x\|$ for each $x \in X$ (for some $\delta>0)$. As in the previous proofs we set $(x, y)=f_{x}(y)$ and we get easily that it is a positive definite sesquilinear form. Now

$$
|x|^{2}=f_{x}(x) \leq\left\|f_{x}\right\|\||x\|\leq\| F|\||| x \|^{2}
$$

To prove the reverse inequality, we need to show previously the surjectivity of $F$. It is a straightforward consequence of the reflexivity of $X: \operatorname{RanF}$ is a dense (and closed) subspace of $X^{*}$ because $R a n F^{\perp}$ (polar space of $R a n F$ ) is the null space, as one can easily check. Now, let $x \in X$ and $f \in X^{*}$, with $\|f\|=1$, such that $\|x\|=f(x)$. By the surjectivity of $F$, we have $f=f_{y}$, for a unique $y \in X$. So (using the Cauchy-Schwarz inequality on the positive definite form $(\cdot, \cdot)$ )

$$
\|x\|=f_{y}(x)=(y, x) \leq|y||x| \leq|| F||^{1 / 2}|| y|||x| \leq\left.\delta^{-1}|| F\right|^{1 / 2}|x|
$$

This ends the proof.

Remark 6. The assumption about the reflexivity of $X$ is, in some sense, necessary. Indeed a straightforward application of James' characterization of reflexivity shows that if $X$ is a real Banach space which is isometrically isomorphic to its dual via $x \rightarrow f_{x}$ and this isomorphism is such that $\overline{f_{x}(y)}=f_{y}(x)$, then $X$ is reflexive (see for instance [Li70]).

\section{Contraction of a Banach space into a Hilbert space}

Theorem 5 suggests an observation that might be of interest. Indeed, we have used the fact that RanF is closed and the reflexivity of $X$ only to prove that $C|| x|| \leq|x|$. Thus we have the following

Corollary 7. Let $X$ be a normed space and $F: x \in X \rightarrow f_{x} \in X^{*}$ be continuous and verifying the following

(1) $f_{x}(y)=\overline{f_{y}(x)}$

(2) $x \in \operatorname{Ker}\left(f_{x}\right) \Rightarrow x=0$

Then $(x, y)=f_{x}(y)$ defines a pre-Hilbertian structure on $X$ and the topology induced by $(\cdot, \cdot)$ is weaker than norm-topology.

Let us to denote $\widetilde{X}$ for the completion of $X$ with respect to the inner product $f_{x}(y)$. Let us calculate $\tilde{X}$ in some simple cases. 
(1) We consider the contraction of $l^{1}$ into its dual $l^{\infty}$ given by the "identity". It is easy to check the $\widetilde{l^{1}}=l^{2}$.

(2) Let $L^{1}(B(H))$ and $L^{2}(B(H))$ respectively the trace class and the Hilbert-Schmidt operator on a Hilbert space $H . L^{1}(B(H))$ is canonically embedded into its dual $B(H)$ through the conjugate-linear map $T \rightarrow \operatorname{tr}\left(T^{*} \cdot\right)$. Thus $\widetilde{L^{1}(B(H))}=L^{2}(B(H))$.

In both these examples, a Banach space turns to be contracted into a Hilbert space. The contraction of a Banach space into a Hilbert space is not something special, at least when the space is separable. Indeed the classical Banach-Mazur representation theorem provides an isometry from every separable Banach space into $C[0,1]$, which is obviously contracted into $L^{2}[0,1]$. On the other hand, it is not clear what happens when the space is not separable: one can still apply the BanachMazur theorem to obtain an isometry from $X$ onto a closed subspace of $C\left(X_{1}^{*}\right)$, where $X_{1}^{*}$ stands for the weak* closed unit ball in the dual space of $X$. When does $C\left(X_{1}^{*}\right)$ embed into $L^{2}\left(X_{1}^{*}\right)$ ? To obtain the canonical embedding we need a positive Borel measure whose support is the whole $X_{1}^{*}$, but it is clear that such a measure might not exist. For instance, let $H$ be a non-separable Hilbert space, $\left\{e_{a}, a \in A\right\}$ an orthonormal basis for $H$ and $X=\{x \in H:\|x\| \leq 1\}$ with the weak topology. We set $U_{a}=\left\{x \in X:\left|\left(x, e_{a}\right)\right|^{2}>\frac{1}{2}\right\}$. This is a non countable family of non empty $\left(e_{a} \in U_{a} !\right)$ disjoint (by Parseval!) open (because the functionals $x \rightarrow\left|\left(x, e_{a}\right)\right|^{2}$ are continuous with respect the weak topology) set. Thus, if $\mu$ is a Borel measure on $X$, there exists $a \in A$ such that $\mu\left(U_{a}\right)=0$ and thus $\operatorname{supp}(\mu) \subseteq U_{a}^{c}$.

\section{REFERENCES}

[Al-Be97] Alonso, J. and Benitez, C, Area orthogonality in linear normed space, Arch. Math. 68 (1997), 70-76. [Bi35] Birkhoff, G. Orthogonality in linear metric spaces, Duke Math. J. 1 (1935), 169-172.

[Di83] Diminnie, C.R. A new orthogonality relation for normed linear spaces, Math. Nachr. 114 (1983), 192-203.

[Dr-Ya05] Drivaliaris, D. and Yannakakis, N. Hilbert space structure and positive operators, J. Math. Anal. Appl. 305 (2005), 560-565.

[Ja47] James, R.C. Orthogonality and linear functionals on normed linear spaces, Trans. Amer. Math. Soc. 61 (1947), 265-292.

[Ja45] James, R.C. Orthogonality in normed linear spaces, Duke. Math. J. 12 (1945), 291-302.

[Le78] Leichtweiss, K. Zur Expliziten Bestimmung der Norm der Seldadjungierten Minkowski Raume, Res. Math. 1 (1978) 61-87.

[Li70] Lin, B.L. On Banach spaces isomorphic to its conjugate, Math. Res. Center, Nat. Taiwan Univ., Taipei (1970) 151-156.

[Li-Tz71] Lindenstrauss, J. and Tzafriri, L. On the complemented subspaces problem, Israel J. Math. 9 (2) (1971) 263-269.

[Pa86a] Partington, J.R. Self-conjugate polyhedral Banach spaces, Bull. London Math. Soc. 18 (1986) 284-286

[Pa86b] Partington, J.R. Orthogonality in normed spaces, Bull. Austral. Amer. Soc. vol. 33 (1986), 449-455.

[Sz-Za81] Sztencel R. and Zaremba P. On self-conjugate Banach spaces, Colloq. Math. 44 (1981), 111-115.

University of Neuchatel, SWitZERlAND

E-mail address: valerio.capraro@unine.ch

UNIVERSity OF Rome, LA SAPIENZA, ITALY

E-mail address: s-rossi@mat.uniroma1.it 